OPEN ACCESS

Edited by:

Sabine Levi,

Institut National de la Santé et de la Recherche Médicale (INSERM),

France

Reviewed by: James Ames, University of California, Davis, United States

Chuang Wang,

Ningbo University, China

*Correspondence:

Selva Rivas-Arancibia

srivas@unam.mx

José M. Saniger

jose.saniger@ccadet.unam.mx

Received: 19 January 2017 Accepted: 21 April 2017

Published: 22 May 2017

Citation:

Rivas-Arancibia S,

Rodríguez-Martínez E,

Badillo-Ramírez l, López-González U and Saniger JM (2017) Structural

Changes of Amyloid Beta in Hippocampus of Rats Exposed to Ozone: A Raman Spectroscopy

Study.

Front. Mol. Neurosci. 10:137. doi: 10.3389/fnmol.2017.00137

\section{Structural Changes of Amyloid Beta in Hippocampus of Rats Exposed to Ozone: A Raman Spectroscopy Study}

\author{
Selva Rivas-Arancibia ${ }^{1 *}$, Erika Rodríguez-Martínez ${ }^{1}$, Isidro Badillo-Ramírez ${ }^{2}$, \\ Ulises López-González ${ }^{2}$ and José M. Saniger ${ }^{2 *}$
}

\begin{abstract}
'Departamento de Fisiología, Facultad de Medicina, Universidad Nacional Autónoma de México, Ciudad de México, Mexico, ${ }^{2}$ Centro de Ciencias Aplicadas y Desarrollo Tecnológico, Universidad Nacional Autónoma de México, Ciudad de México, Mexico
\end{abstract}

The aim of this work was to study the effect of oxidative stress on the structural changes of the secondary peptide structure of amyloid beta 1-42 (A $\beta$ 1-42), in the dentate gyrus of hippocampus of rats exposed to low doses of ozone. The animals were exposed to ozone-free air (control group) and 0.25 ppm ozone during 7, 15, 30, 60, and 90 days, respectively. The samples were studied by: (1) Raman spectroscopy to detect the global conformational changes in peptides with $\alpha$-helix and $\beta$-sheet secondary structure, following the deconvolution profile of the amide I band; and (2) immunohistochemistry against $A \beta$ 1-42. The results of the deconvolutions of the amide I band indicate that, ozone exposure causes a progressively decrease in the abundance percentage of $\alpha$-helix secondary structure. Furthermore, the $\beta$-sheet secondary structure increases its abundance percentage. After 60 days of ozone exposure, the $\beta$-sheet band is identified in a similar wavenumber of the $A \beta 1-42$ peptide standard. Immunohistochemistry assays show an increase of A $\beta$ 1-42 immunoreactivity, coinciding with the conformational changes observed in the Raman spectroscopy of A $\beta$ 1-42 at 60 and 90 days. In conclusion, oxidative stress produces changes in the folding process of amyloid beta peptide structure in the dentate gyrus, leading to its conformational change in a final $\beta$-sheet structure. This is associated to an increase in $A \beta 1-42$ expression, similar to the one that happens in the brain of Alzheimer's Disease (AD) patients.

Keywords: oxidative stress, Raman spectroscopy, amyloid beta 1-42, ozone, neurodegeneration, Alzheimer's disease

\section{INTRODUCTION}

Environmental pollution has become a public health issue in densely populated cities. One of the main pollutants resulting from photochemical air pollution is ozone. There is a large number of evidences that show the role played by ozone air pollution and its association to neurodegenerative diseases (Block and Calderón-Garcidueñas, 2009; Mumaw et al., 2016) such as Alzheimer's Disease (AD; Moulton and Yang, 2012; Jung et al., 2015).

Exposition to ozone induces a state of oxidative stress if it is breathed chronically (RivasArancibia et al., 2010). In previous works conducted in our laboratory, we have reported a non-invasive model of progressive neurodegeneration in rat hippocampus, caused by oxidative stress induced by low doses of ozone (Rivas-Arancibia et al., 2010; Rodríguez-Martínez et al., 2016). 
Using this model in healthy rats, we have demonstrated that, without any other additional factors, oxidative stress by itself can produce damage and death cell along with the formation of $A \beta$ 1-42, similar to what happens in AD patients (Rivas Arancibia et al., 2011; Hernández-Zimbrón and Rivas-Arancibia, 2014). In addition, ozone exposure also leads to a deficit in the learning and memory processes as well as motor and behavioral impairment in rats (Rivas-Arancibia et al., 1998; Dorado-Martínez et al., 2001).

The presence of $A \beta 1-42$ in the brain of $A D$ patients is the final outcome of a series of metabolic changes that take place throughout extended periods of time during which the state of oxidative stress plays a key role. Replicating these changes in an animal model allows us to study the development of conformational alterations in peptides and proteins that occur during a chronic process of progressive neurodegeneration.

The peptide $A \beta$ is generated by the cleavage of the amyloid precursor protein (APP) with the aid of $\alpha$ - and $\beta$-enzymes and $\gamma$-secretase (Hernández-Zimbrón and Rivas-Arancibia, 2014). The breakdown of APP occurs in two pathways: the non-amyloidogenic and amyloidogenic one. The amyloidogenic pathway is an alternative breakdown pathway of APP that leads to $A \beta$ formation. The $A \beta$ peptide of 40 amino acids $(A \beta$ 1-40) is the most common (90\%), while the $10 \%$ usually corresponds to the full-length $A \beta$ 1-42. The latter is more hydrophobic and easily induces fibril formation.

Recent solid state NMR (ssNMR) studies have shed new light on A $\beta$ 1-42 structure at atomic resolution (Xiao et al., 2015). They showed that triple parallel- $\beta$-sheet segments form the $A \beta$ $1-42$ with $\beta$-sheet motif, while Ahmed et al. (2010) proposed a mechanism to explain the conversion of $A \beta$ 1-42 into fibrils. In addition, several molecular dynamics simulations have been performed in order to elucidate a possible mechanism in the conformational dynamics of $\mathrm{A} \beta$ 1-42. For example in different conditions, $A \beta$ can aggregate in the form of monomers to oligos and finally in cytotoxic protofilaments of $A \beta$ 1-42. In addition, there are studies that show the specific amino acid regions that favor the $\beta$-sheet assembly into insoluble protofilaments, in order to correlate these results with those obtained with NMR and X-ray diffraction experiments (Buchete et al., 2005; Zhu et al., 2012; Lee et al., 2016). However, neither the molecular mechanism nor the different structural conformations that $\mathrm{A} \beta$ $1-42$ adopts, from the monomer phase to its final aggregation in insoluble protofibrils, have been established in animal models.

On the other hand, Raman spectroscopy has played an important role in the study of the influence of microenvironment on the biomolecular conformation, due to its ability to record the vibrational spectra of molecules in their biological environment even in animal models.

Even though, Raman spectroscopy cannot determine the molecular conformation of complex biomolecules as peptides or proteins at atomic level, it is highly informative for tracing the global conformational changes induced by modifications of its microenvironment. In general, the interpretation of the Raman results of biomolecules is based on the establishment of empirical correlations derived from structural studies performed with other techniques that provide information at atomic level, such as ssNMR, X-ray crystallography and molecular dynamics simulation (Zhang et al., 2000; Barth and Zscherp, 2002; Kong and $\mathrm{Yu}, 2007)$.

It is well known that Raman spectral intervals, known as amide regions, provide information on the changes that occur in the secondary structure of peptides and proteins (Uzunbajakava et al., 2003; Ruiz-Chica et al., 2004; Movasaghi et al., 2007; Bonnier and Byrne, 2012). In a normal Raman spectrum, there are three main regions that provide potential information on the vibrational modes of the proteins: amide I, II and III. Amide I band (1600-1700 $\left.\mathrm{cm}^{-1}\right)$, containing the in-plane vibrational mode of the $\mathrm{C}=\mathrm{O}$ stretching and the $\mathrm{N}-\mathrm{H}$ bending of the polypeptide bond, these modes are frequently used to follow the evolution of the peptide secondary conformational change. This band is mostly originated by vibrations from the amide groups found along the polypeptide skeleton, which are also sensitive to the $\Phi$ and $\psi$ angles of each residue as well as the H-bonding pattern and peptide-peptide dipole coupling. The vibration of the amide modes is sensitive to the conformational changes of the polypeptide skeleton; it allows tracking the structural variations, which may be taking place in peptides and proteins during a progressive neurodegeneration process.

In addition, Raman spectroscopy has been reported in the literature as a tool to obtain information on biomolecules in the tissue of AD patients (Sudworth and Krasner, 2004; Chen et al., 2009; Tay et al., 2011; Park et al., 2013). These studies focus primarily on the exploration of the molecular conformation of insoluble $\mathrm{A} \beta$ 1-42 already formed, either chemically synthesized or obtained from patients with $\mathrm{AD}$ brains. However, to the best of our knowledge, there are no reports on the conformational changes that take place during the formation of $A \beta 1-42$ through the amyloidogenic pathway along the development of the progressive neurodegeneration process in an animal model.

In the literature, there are few works focusing on the conformational change of $A \beta$ 1-42 under oxidative conditions. Some experiments have shown that methionine residues are very sensitive to oxidation, favoring a random coil structure in water at pH 4 (Watson et al., 1998; Friedemann et al., 2015). Some other reports have provided evidence that lipid oxidation favors the fibril aggregation of $A \beta$ 1-42 into a $\beta$-sheet conformation (Boyd-Kimball et al., 2004; Butterfield et al., 2013; Korshavn et al., 2017). However, those results are not enough to establish a clear correlation to explain the steps followed during the conformational change of $\mathrm{A} \beta \mathrm{1-42}$ in the $\mathrm{AD}$, under oxidizing conditions.

The aim of this work is to study the effect of oxidative stress, caused by exposure to low doses of ozone, over the conformational changes of the secondary structure of $\mathrm{A} \beta$ 1-42, in the dentate gyrus of rat hippocampus in a model of progressive neurodegeneration.

\section{EXPERIMENTAL PROCEDURE}

\section{General Procedure}

Animal care and handling were conducted in accordance with the National Institute of Health Guidelines for Animal Treatment, 
the Norma Oficial Mexicana NOM-036-SSA2-2002, and were approved by the Ethics Committee of the Medicine School at the National Autonomous University of Mexico.

Seventy-two male Wistar rats weighing 250-300 g were individually housed in acrylic boxes with free access to water and food (Purina, Minnetonka, MN, USA) and kept in a clear-air room. They were randomly divided into six experimental groups ( $n=12$ each): Group 1, exposed to an air stream free of ozone during 30 days; Group 2, exposed for 7 days to ozone; Group 3, exposed for 15 days to ozone; Group 4, exposed for 30 days to ozone; Group 5, exposed for 60 days to ozone; and Group 6, exposed for 90 days to ozone. The rats were exposed to ozone $(0.25 \mathrm{ppm})$ daily for $4 \mathrm{~h}$. Immediately after ozone exposure, the animals were returned to their home cages, and $2 \mathrm{~h}$ later they were processed for the following techniques: (1) Raman spectroscopy to follow the conformational structural changes of the $A \beta$ peptides; and (2) immunohistochemistry $A \beta$ $1-42$.

\section{Ozone Exposure}

Animals were put inside a chamber with a diffuser connected to a variable flux ozone generator $(5 \mathrm{l} / \mathrm{s})$ daily for $4 \mathrm{~h}$. The procedure used has been described in other works (Pereyra-Muñoz et al., 2006; Rivas-Arancibia et al., 2010). The air feeding the ozone converter was filtered and purified. Ozone production levels were proportional to the current intensity and to the airflow. A PCI Ozone and Control System Monitor (West Caldwell, NJ, USA) was used to measure the ozone concentration inside the chamber throughout the experiment and to keep a constant ozone concentration.

\section{Air Exposure}

The control group was treated in the same chamber described above using a flow of ozone-free purified air.

\section{Preparation of Tissue Samples for Raman Spectroscopy}

After the last exposure to ozone, the rats were deeply anesthetized using pentobarbital sodium $(50 \mathrm{ml} / \mathrm{kg})$. The brains were extracted, perfused with $4 \%$ paraformaldehyde, dehydrated, and embedded in paraffin. Afterwards, $20 \mu \mathrm{m}$ slices were cut using a microtome (SHUR/Cut2500, General Data Healthcare). The sections were deparaffinized and hydrated before conducting the spectroscopic analysis.

\section{Raman Spectroscopy Procedure}

The deparaffinized sections were first examined by light microscopy to define a study region where six different cells were randomly selected. Five single spectra were recorded for each selected cell at different cytoplasm points close to the membrane cell. In this way, we obtained 30 single Raman spectra for each section.

Raman spectra were obtained using a Witec Alpha300 R confocal microscopy system, with a laser excitation source of $532 \mathrm{~nm}$ at a power of $0.7 \mathrm{~mW}, 600 \mathrm{l} / \mathrm{mm}$ grating, and a $50 \times$ plane Fluor objective with a numerical aperture of 0.45 at a working distance of $4.5 \mathrm{~mm}$. The spectral resolution used was $4 \mathrm{~cm}^{-1}$ while a $576 \times 400$ pixel thermoelectric-cooled charge-coupled device (CCD) was used as detector.

Before each measurement, a system calibration was performed using a silicon chip in order to check the standard band position and the intensity. Data were measured over the $200-4000 \mathrm{~cm}^{-1}$ spectral range. Each spectrum consisted of five accumulations with an integration time of $0.5 \mathrm{~s}$ to avoid tissue damage. As a reference, we used pure A $\beta$ 1-42 (SigmaAldrich) to obtain its Raman spectra under similar experimental conditions.

\section{Spectral Analysis}

The spectra were pre-processed for base line correction and cosmic ray removal using WITEC Project 2.0 software. After that, the 30 spectra obtained from each tissue section under study were vector-normalized with Wolfram-Mathematica software and, finally, each set of 30 spectra was averaged. This procedure was used for the control group as well as the groups exposed to ozone for 7, 15, 30, 60 and 90 days.

Additionally, a deconvolution of the amide I band $\left(1600-1700 \mathrm{~cm}^{-1}\right)$ of the spectra was performed in order to visualize the subjacent sub-bands that compose the whole complex amide I band. The original spectra were baselinecorrected before the deconvolution analysis and smoothed with the Savitzky-Golay (SG) filter with five-point window. The deconvolution was performed using Origin 6.0 software, selecting the Gaussian peak type option. The number of sub-bands employed for this spectral analysis was defined by the second derivative of the whole amide I band. In addition, two bands at $\sim 1604 \mathrm{~cm}^{-1}$ and $\sim 1612 \mathrm{~cm}^{-1}$ were included with the amide I band during the deconvolution process since they were not baseline separated from the amide I feature. The values of the area under the curve of each sub-band were taken, in a first approximation, as proportional to the relative abundances of the peptide motifs associated with each sub-band.

\section{Immunohistochemistry Technique}

Six animals from each group were anesthetized with pentobarbital sodium $(50 \mathrm{mg} / \mathrm{kg}$ i.p.; Sedalpharma, Edo. de México, Mexico) and transcardially perfused with $4 \%$ paraformaldehyde (Sigma-Aldrich Chemie, Germany) in $0.1 \mathrm{M}$ phosphate buffer (J.T Bakker E.U., New Jersey, Kentucky and Mexico; PB, Tecsiquim; pH 7.4). The brains were post fixed with $10 \%$ formaldehyde (J.T Bakker U.S.A., New Jersey, Kentucky and Mexico) for $24 \mathrm{~h}$ and embedded in paraffin (McCormick, St. Louis, MO, USA). Sagittal slices of the brain containing the hippocampi were cut at $5 \mu \mathrm{m}$ on a microtome (ShurCut 2500. General Data Healthcare) and mounted on slides. Slices of each brain containing the hippocampi were deparaffinized and pretreated with heat retrieval solution (Biocare Medical, Concord CA, USA) and introduced into an electric pressure cooker (Decloacking Chamber, Biocare Medical) for $20 \mathrm{~min}$. After being washed with distilled water and treated with $3 \%$ hydrogen peroxide (J.T Baker U.S.A., New Jersey, Kentucky and Mexico) for 5 min, the slices were rinsed again and treated with a blocking reagent (Background Sniper, Biocare Medical, Concord, CA, USA) for $10 \mathrm{~min}$. 
They were washed with saline phosphate buffer (PBS, Merck, Germany) and incubated overnight at $4^{\circ} \mathrm{C}$ with $\mathrm{A} \beta$ 1-42 (purified monoclonal mouse antibody, diluted 1:50 (ANASPEC, Fremont, CA, USA). Sections were rinsed with PBS and treated with secondary antibody using Trekkie Universal Link (Starr Trek Universal HRP Detection; Biocare Medical) for $1 \mathrm{~h}$. The sections were later washed with PBS, and then treated with Trekavidin-HRP Label (Starr Trek Universal HRP Detection, Biocare Medical) for $30 \mathrm{~min}$. Bound antibody was visualized using 3,3'-Diaminobenzidine (DAB Substrate Kit, ScyTek, Logan, UT, USA) as the chromogen. The slices were washed with distilled water and contrasted with hematoxylin buffer solution (Concord, CA, USA). Representative brain slices from each group were processed in parallel. Following cover slipping with Entellan (F/550 ml Merck, Germany), the slices were examined with an Olympus BX41 Microscope (Japan) and photographed (Evolution VF-F-CLR-12 Media Cybernetics camera; Bethesda, MD, USA).

\section{RESULTS}

\section{Raman Spectra of Rat Hippocampus Tissue}

The Raman spectra of the "finger-print" region $\left(600-1800 \mathrm{~cm}^{-1}\right)$ of rat hippocampus tissue were analyzed (Figure 1). The spectra presented correspond to the control groups (Figure 1a) and the

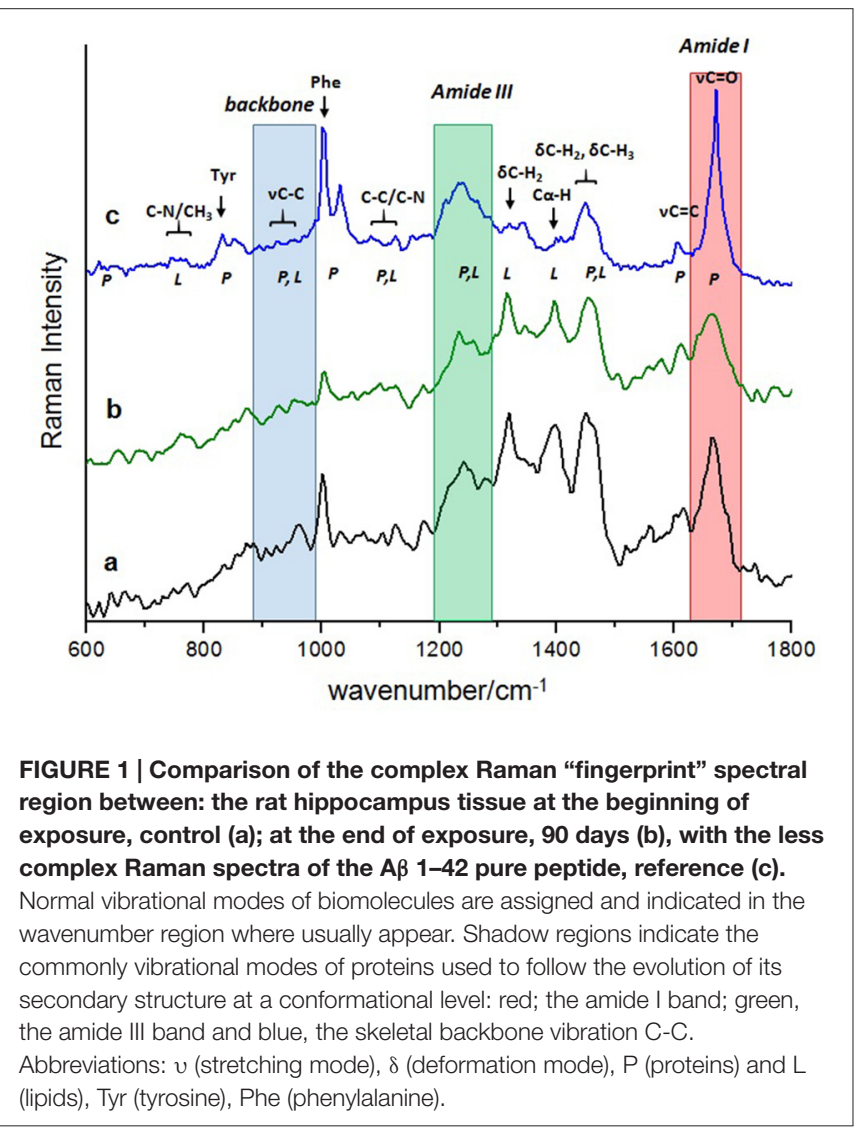

group exposed to ozone for 90 days (Figure 1b), together with that of the pure $A \beta$ 1-42 peptide used as a reference (Figure 1c). The complexity of the Raman spectra, both from a tissue ( $a$ and $b$ ) and from a pure peptide sample (c), is seen in a comparative way in Figure 1.

The regions conventionally used to make a structural study of proteins based on their Raman spectrum are amide I, amide III and skeletal backbone vibration $\mathrm{C}-\mathrm{C}$, which are shaded in Figure 1. It is important to note that the amide band I (1640-1680 $\left.\mathrm{cm}^{-1}\right)$ of each spectrum presents a different profile in relation to bandwidth, wavenumber and intensity. From a direct observation of this band, it is not possible to estimate the dominant secondary conformation in each case. To achieve this purpose, the profile of the amide I band must be mathematically processed by a deconvolution analysis to obtain this information.

The regions of amide III $\left(1200-1340 \mathrm{~cm}^{-1}\right)$ and skeletal backbone vibration C-C $\left(890-980 \mathrm{~cm}^{-1}\right)$ help, in principle, to corroborate the secondary structure derived from the analysis of the amide I band. However, many other vibrational modes are also in this region, which come from other biomolecules that are present in the rat tissue and overlap with each other. This makes it difficult, in our case, to use these regions (amide III and skeletal backbone vibration $\mathrm{C}-\mathrm{C}$ ) to confirm the secondary structure of the $A \beta 1-42$ in our results.

\section{Raman Spectrum of Reference $A \beta$ 1-42 Peptide}

The Raman spectrum of the commercial $A \beta 1-42$ peptide that is used as a reference for the experimental direct identification

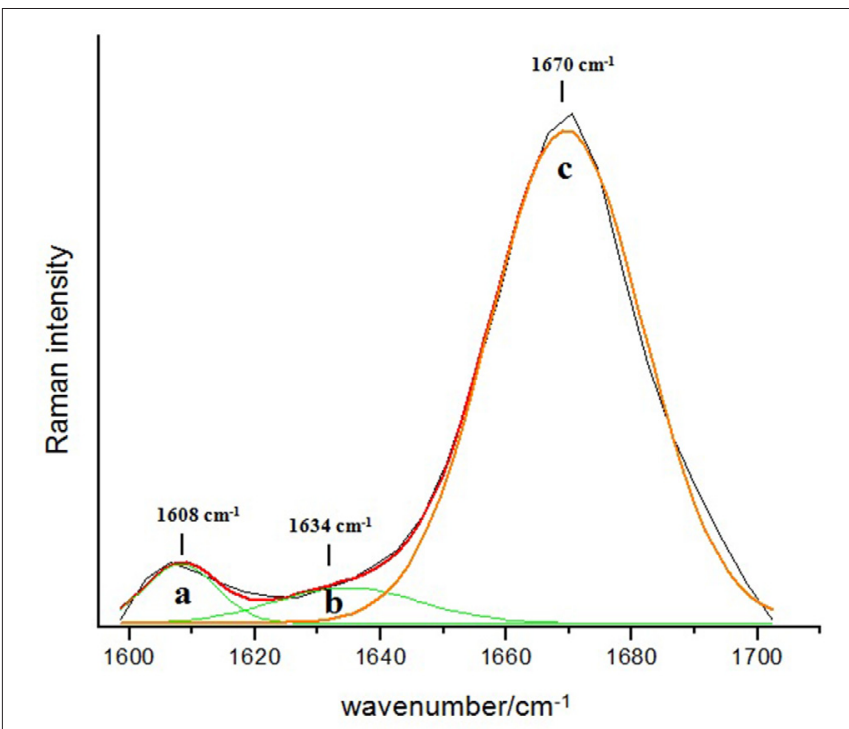

FIGURE 2 | Deconvolution of the amide I band region (1600-1700 cm-1) in the Raman spectrum of the reference $A \beta$ 1-42. The dominant band at $1670 \mathrm{~cm}^{-1}$ with $89 \%$ of relative abundance (c) indicates the presence of the $\beta$-sheet secondary structure type, while the two minor bands at $1608 \mathrm{~cm}^{-1}$ (a) and $1634 \mathrm{~cm}^{-1}$ (b) are assigned to the amino acids side chains (6\%) and the aromatic amino acids ring mode (5\%), respectively. 
of the $\beta$-sheet conformation in the amide I band of this peptide is shown in Figure 2. The spectrum is dominated by a Gaussian type band centered at $1670 \mathrm{~cm}^{-1}$ (Figure 2c) which is assigned mainly to the $\mathrm{C}=\mathrm{O}$ stretching vibration of the $\beta$-sheet secondary structure in the A $\beta$ 1-42 peptide (Barth and Zscherp, 2002; Rygula et al., 2013). Additionally, the deconvolution of the spectral region between $1600 \mathrm{~cm}^{-1}$ and $1700 \mathrm{~cm}^{-1}$ shows two minor bands: $1608 \mathrm{~cm}^{-1}$ (Figure 2a) and $1634 \mathrm{~cm}^{-1}$ (Figure 2b), which are assigned to the amino acid side chains and the aromatic amino acid ring mode vibration, respectively (Rahmelow et al., 1998; Rygula et al., 2013). The percentage of area under the curve for each of these bands is $89 \%$ for the $\beta$-sheet structure, $6 \%$ for the amino acid side chains and 5\% for the aromatic amino acid ring mode vibration.

\section{Raman Spectra of Tissue from the Different Rat Groups, Control and Those Exposed to Different Periods of Ozone}

Confocal micrographs of hippocampal cells where the Raman spectra were obtained (Figure 3, columns A and B) along with the results from the mathematical deconvolutions of the amide I band (Figure 3, column C). The micrographs of the immunohistochemistry against $A \beta$ 1-42 for each of the experimental groups (control and those exposed to ozone for $7,15,30,60$ and 90 days) are also shown (Figure 3, column D).

The deconvolution of the amide I band (Figures 3C1-C6) for the groups evidences the presence of vibrational modes assigned as follows: $1602-1610 \mathrm{~cm}^{-1}$ amino acid side chains vibrations (Rahmelow et al., 1998), 1615-1635 $\mathrm{cm}^{-1}$ aromatic amino acid ring mode vibrations (Rygula et al., 2013), 1644-1650 $\mathrm{cm}^{-1}$ random coil structure (Sekhar et al., 2015), 1655-1668 $\mathrm{cm}^{-1}$ $\alpha$-helix secondary structure (Lippert et al., 1976; Rygula et al., 2013; Kurouski et al., 2015), 1670-1682 $\mathrm{cm}^{-1} \beta$-sheet secondary structure (Mahadevan-Jansen and Richards-Kortum, 1996; Wen, 2007; Kurouski et al., 2015), and 1683-1685 cm-1 $\beta$-turn structural motifs (Barth and Zscherp, 2002). Table 1 summarizes the distribution of the sub-bands resulting from the deconvolution analysis for the control and all the ozone-exposed groups, indicating the percentage relative abundance and the assignment for each Raman vibrational mode.

In the control group the amide I band (Figure 3C1), is mostly composed by the corresponding $\alpha$-helix peptide secondary structure (60\%) (band 4), this band is centered at $1665 \mathrm{~cm}^{-1}$. Additionally, there is a minor contribution of the vibrational motifs of the amino acids side chain (10\%) (band 1), the aromatic amino acids ring mode (band 2) (5\%), the unordered structure (10\%) (band 3 ) and the $\beta$-turn type structure (15\%) (band 5). On the other hand, the micrograph of the dentate gyrus shows absence of reactivity to $\mathrm{A} \beta \quad 1-42$ by immunohistochemistry (Figure 3D1).

The deconvolution of the amide I band for the groups exposed to ozone for 7 days (Figure 3C2), this band still shows a clear majority of the $\alpha$-helix secondary structure conformation (47\%) now centered at $1668 \mathrm{~cm}^{-1}$ (band 4). Additionally, there is an increase in the abundance of the bands corresponding to the unordered structure (33\%) (band 3), the vibrational modes of amino acids side chain (8\%) (band 1), and the $\beta$-turn structure (12\%) (band 5). For its part, the immunohistochemistry assay shows absence of immunoreactivity against $\mathrm{A} \beta$ 1-42 (Figure 3D2).

At 15 days of ozone exposure (Figure 3C3), there is an evident change in the amide I band profile, particularly in the region of the high wavenumbers where the formation of a shoulder at $1680 \mathrm{~cm}^{-1}$ is observed (band 5); these changes are reflected in the deconvolution sub-bands. The predominant band is still the one corresponding to the $\alpha$-helix secondary structure (45\%), centered now at $1662 \mathrm{~cm}^{-1}$ (band 4), together with the vibrations of the amino acids side chain (9\%) (band 1) and the aromatic amino acid ring mode (13\%) (band 2). The most significant change for this period of exposure is observed in the band associated to the $\beta$-turn structure (band 5), which increases its relative abundance (16\%) and moves towards slightly lower wave numbers centering at $1681 \mathrm{~cm}^{-1}$. For its part, in the microphotograph of the dentate gyrus in the hippocampus, the beginning of a diffuse extracellular immunoreactivity for $\mathrm{A} \beta$ 1-42 is observed (Figure 3D3).

In the group exposed to ozone for 30 days (Figure 3C4), the amide I band profile still shows the change in its profile for the high wavenumber side. The corresponding deconvolution now shows four sub-bands: one associated to the aromatic amino acids ring mode (6\%) (band 2), the unordered secondary structure (30\%) (band 3) and the $\alpha$-helix secondary structure (41\%) (band 4 ). The sub-band corresponding to the $\beta$-turn structures increases its abundance (23\%) (band 5) and continues moving toward low wavenumbers. On the other hand, in the corresponding micrograph of the dentate gyrus in the hippocampus, $A \beta$ 1-42 shows a clear increase of intracellular immunoreactivity, together with cell edema and extracellular diffuse immunoreactivity (Figure 3D4).

After 60 days of ozone exposure (Figure 3C5), the changes in the amide I band envelope are even more evident, a fact which is reflected in the relevant changes in the result of its deconvolution. Indeed, the sub-band corresponding to the $\alpha$-helix secondary structure (band 4) considerably decreases its abundance $(22 \%)$ while band 6 becomes predominant (52\%) and is now centered at $1671 \mathrm{~cm}^{-1}$, in clear accordance with the wavenumber of the previously assigned $\beta$-sheet structure of pure $A \beta$ 1-42 presented in Figure 2. In a similarly evident way, the $\beta$-turn band (band 5) shows a significant decrease in its abundance (5\%) and a deviation toward higher wavenumbers $\left(1690 \mathrm{~cm}^{-1}\right)$. For their part, the bands of the amino acids side chains (band 1) and the mode of the aromatic amino acids ring (band 2) remain unchanged. In agreement with these spectral changes, the immunohistochemistry in the dentate gyrus of the hippocampus shows an intracellular increase of $\mathrm{A} \beta$ 1-42 immunoreactivity, which is also evident in the neuropil, together with cell swelling and pyknotic nuclei (Figure 3D5).

The deconvolution of the amide I band for the group exposed to ozone for 90 days (Figure 3C6) shows a similar distribution to that of the previous group. Once again, the most abundant sub-band (48\%) corresponds to the $\beta$-sheet 

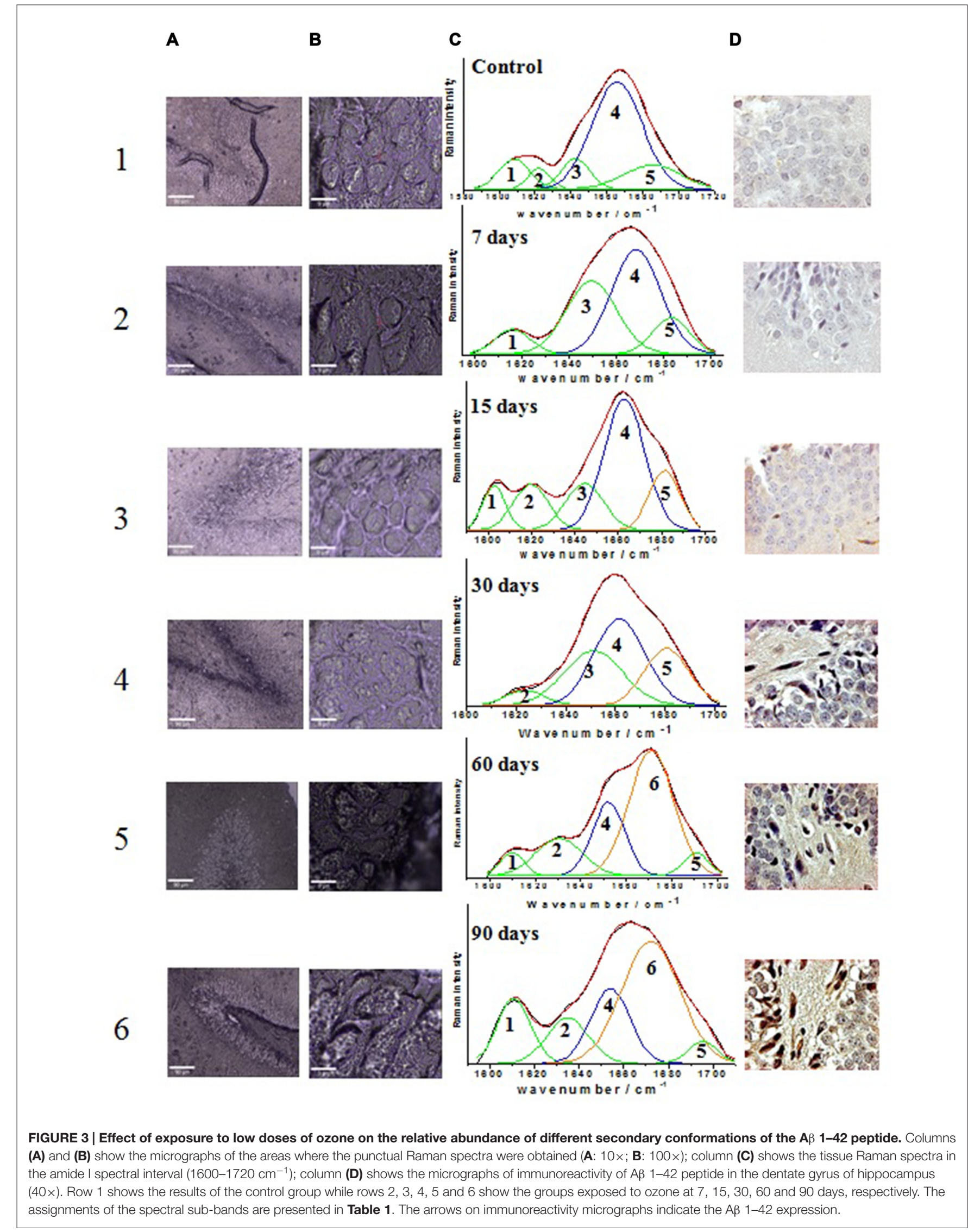

D 
TABLE 1 | Deconvolution of the amide I mode of different proteins and peptides along exposure time.

\begin{tabular}{|c|c|c|c|c|}
\hline $\begin{array}{l}\text { Band } \\
\text { number }\end{array}$ & $\begin{array}{l}\text { Center } \\
\left(\mathrm{cm}^{-1}\right)\end{array}$ & $\begin{array}{l}\text { Band } \\
\text { area }\end{array}$ & $\begin{array}{c}\text { Abundance } \\
\text { (\%) }\end{array}$ & Assignment \\
\hline \multicolumn{5}{|c|}{ Control group, no exposure to ozone } \\
\hline 1 & 1608 & 0.073 & 10 & Amino acids side chains \\
\hline 2 & 1622 & 0.036 & 5 & Aromatic amino acids ring mode \\
\hline 3 & 1642 & 0.069 & 10 & Unordered structure \\
\hline 4 & 1665 & 0.043 & 60 & $\alpha$-helix \\
\hline 5 & 1685 & 0.108 & 15 & $\beta$-turn \\
\hline \multicolumn{5}{|c|}{ Group exposed to ozone for 7 days } \\
\hline 1 & 1615 & 0.068 & 8 & Amino acids side chains \\
\hline 3 & 1649 & 0.292 & 33 & Unordered structure \\
\hline 4 & 1668 & 0.405 & 47 & $\alpha$-helix \\
\hline 5 & 1683 & 0.106 & 12 & $\beta$-turn \\
\hline \multicolumn{5}{|c|}{ Group exposed to ozone for 15 days } \\
\hline 1 & 1602 & 0.078 & 9 & Amino acids side chains \\
\hline 2 & 1619 & 0.124 & 13 & Aromatic amino acids ring mode \\
\hline 3 & 1644 & 0.140 & 16 & Unordered structure \\
\hline 4 & 1662 & 0.412 & 46 & $\alpha$-helix \\
\hline 5 & 1681 & 0.143 & 16 & $\beta$-turn \\
\hline \multicolumn{5}{|c|}{ Group exposed to ozone for 30 days } \\
\hline 2 & 1623 & 0.051 & 6 & Aromatic amino acids ring mode \\
\hline 3 & 1650 & 0.277 & 30 & Unordered structure \\
\hline 4 & 1661 & 0.382 & 41 & $\alpha$-helix \\
\hline 5 & 1680 & 0.218 & 23 & $\beta$-turn \\
\hline \multicolumn{5}{|c|}{ Group exposed to ozone for 60 days } \\
\hline 1 & 1609 & 0.037 & 5 & Amino acids side chains \\
\hline 2 & 1630 & 0.118 & 16 & Aromatic amino acids ring mode \\
\hline 4 & 1652 & 0.160 & 22 & $\alpha$-helix \\
\hline 6 & 1671 & 0.376 & 52 & $\beta$-sheet \\
\hline 5 & 1690 & 0.038 & 5 & $\beta$-turn \\
\hline \multicolumn{5}{|c|}{ Group exposed to ozone for 90 days } \\
\hline 1 & 1610 & 0.143 & 15 & Amino acids side chains \\
\hline 2 & 1635 & 0.125 & 13 & Aromatic amino acids ring mode \\
\hline 4 & 1655 & 0.196 & 20 & $\alpha$-helix \\
\hline 6 & 1672 & 0.047 & 48 & $\beta$-sheet \\
\hline 5 & 1695 & 0.042 & 4 & $\beta$-turn \\
\hline
\end{tabular}

secondary structure (band 6) centered at $1672 \mathrm{~cm}^{-1}$. The representative sub-band of the $\alpha$-helix secondary structure (band 4) at $1655 \mathrm{~cm}^{-1}$, decreases its relative abundance to $20 \%$, while the one of the $\beta$-turn structure (band 5), centered at $1695 \mathrm{~cm}^{-1}$, remains around $4 \%$. Moreover, the sub-bands corresponding to the side chains of aromatic amino acids (band 1) and the breathing mode of the aromatic amino acids ring (band 2) have now a contribution of $15 \%$ and $13 \%$, respectively. Once again, consistent with the spectral results, the micrograph of the dentate gyrus of the hippocampus shows increased $A \beta$ 1-42 immunoreactivity, both intracellularly and at the neuropil, with morphological changes as swelling and pyknotic nuclei (Figure 3D6).

In relation with the optical micrographs of columns A and B in Figure 3, which shows the areas where the punctual Raman spectra were obtained, it is important to note the alteration of the morphology of neurons, in particular of the cell membrane, in correlation with the increase in the time of exposure to ozone. These observations are consistent with the results of the immunohistochemistry against A $\beta$ 1-42 presented in column D of Figure 2.

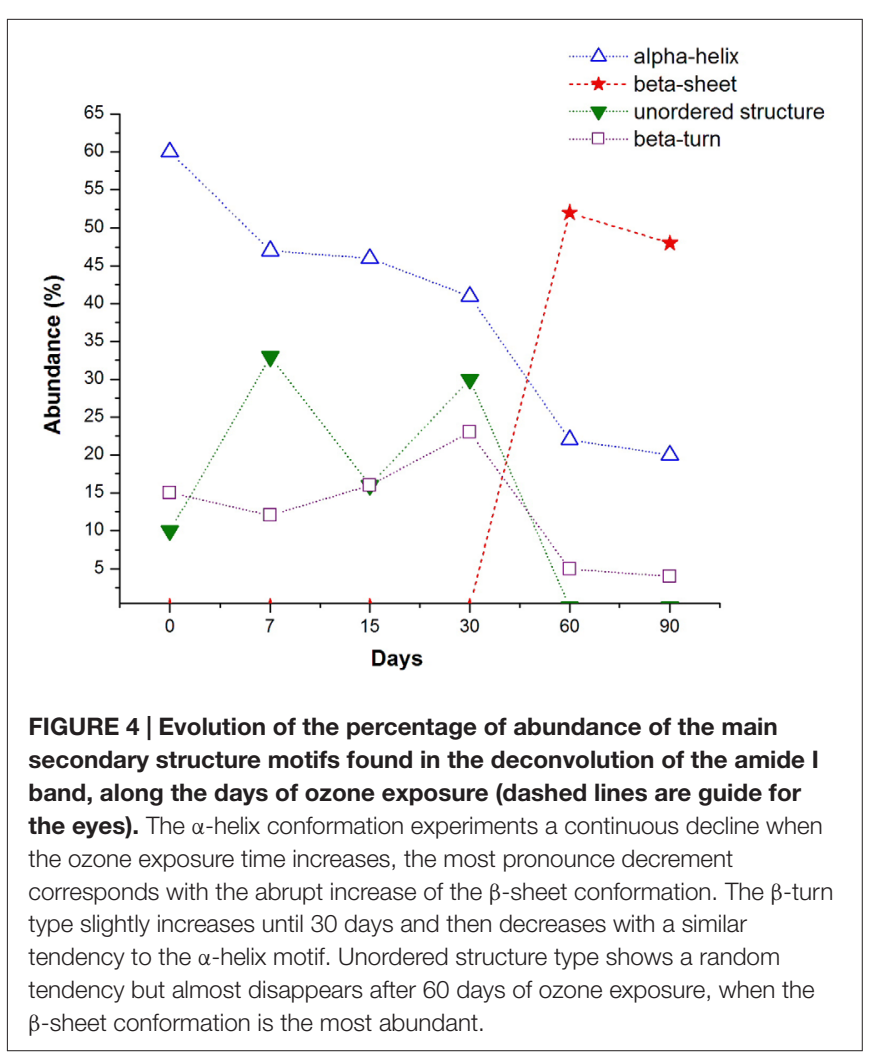

\section{Evolution of the Percentage of Abundance of the Secondary Structure Motifs of the Amide I Band}

Finally, in Figure 4 we show a general view of the trend of the major conformational secondary $A \beta \quad 1-42$ motifs, resulting from the deconvolution analysis of the amide I band of the hippocampal tissue of rats, exposed to low doses of ozone for different periods. It is clear how the $\alpha$-helix motif experiments a continuous decrease as a function of the increase ozone exposure time. The more pronounced decrease of the $\alpha$-helix band between 30 and 60 days of ozone exposure, corresponds with the appearance and the strong increase of the band at $1671 \mathrm{~cm}^{-1}$, associated with the $\beta$-sheet motif of the $A \beta \quad 1-42$ sary structure. After 60 days of ozone exposure, the relative abundance of the $\alpha$-helix and $\beta$-turn motifs seems to reach an almost steady state. On the other hand, there is not a clear trend in the changes of the $\beta$-turn and the non-ordered structures during the first 30 days of exposure to ozone. However, the near disappearance of these bands after 60 days of exposure seems to be correlated with the strong growth of the $\beta$-sheet secondary structure during the same period.

\section{Number of Cells Expressing A $\beta$ 1-42 in Hippocampus of Rats Chronically Exposed to Ozone}

The chronic exposure to low doses of ozone leads to an increase in the number of cells expressing the $A \beta 1-42$ peptide from 30 to 90 days of exposure (Figure 5). Also, we can also observe that the 


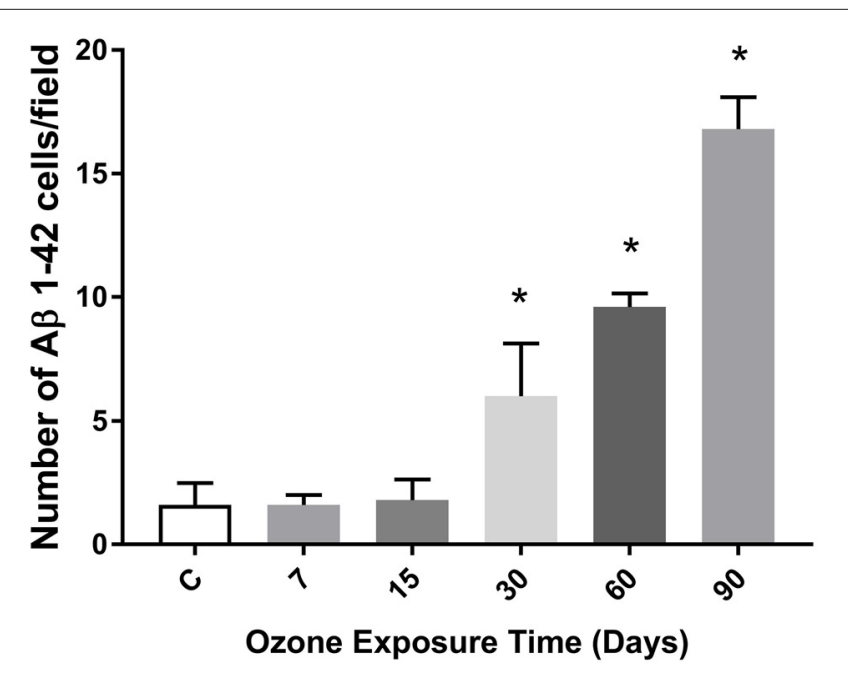

FIGURE 5 | Effects of oxidative stress on the number of immunoreactive cells to $A \beta$ 1-42 depending of the time of exposure to ozone in the hippocampus. The graph shows an increase of the cell number positive to $A \beta 1-42$ at 30,60 and 90 days of ozone treatment $\left({ }^{*} p<0.05\right)$.

average number of cells immunoreactive to beta-amyloid 1-42, increases in accordance with the increase of the abundance of the beta-sheet structure from 30 to 60 days, presented previously in Figure 4.

\section{DISCUSSION}

The Raman spectroscopy results presented in this work, show a temporal evolution of the different structural motifs, mainly $\alpha$-helix and $\beta$-sheet, that conform the secondary structure of the A $\beta$ 1-42 in hippocampus of rats exposed to low doses of ozone for different periods of time.

To the best of our knowledge, these results report, for the first time, the effect of chronic oxidative stress on the conformational secondary structure of $A \beta 1-42$ of hippocampal tissues of rats exposed to low doses of ozone. A predominance of $\alpha$-helix structures in the hippocampus of control animals is clearly established as the initial condition. As the neurodegeneration process advances, from the initial step until 30 days, the $\alpha$-helix structure seems to be destabilized in favor of $\beta$-turn and unordered structures. Subsequently, between 30 and 60 days, when the process of neurodegeneration has set in, the $\beta$-sheet secondary structure is observed to appear and grow at the expense of the $\beta$-turn and unordered structural motifs. Finally, after 60 days of ozone exposure, the $\beta$-sheet motif remains the predominant secondary structure in the $A \beta$ 1-42 peptide. The formed scenario is reinforced by immunohistochemistry studies of hippocampal tissue slices of animals that were treated with low doses of ozone for 60 and 90 days, showing high immunoreactivity against $\mathrm{A} \beta \mathrm{1}-42$.

As described above, we tracked the evolution of the sub-bands that create the amide I spectral interval and its association to the immunohistochemistry response toward $A \beta$ 1-42. This allowed us to distinguish different stages in the temporal evolution of the existing sub-bands in the tissue of the dentate gyrus in the hippocampus of rats exposed to low doses of ozone (Figure 3).

The first stage covers the groups exposed to ozone for 7 and 15 days. The Raman spectra of this stage show a decrease of the sub-band corresponding to the conformation of $\alpha$-helix along with a considerable variability of the sub-bands of unordered and $\beta$-turn structural motifs (Figure 4). All this suggests the beginning of the destabilization of the $\alpha$-helix structure that, in consequence, gives way to other secondary structural motifs.

This first stage may be considered to represent an initial process of the conformational changes, even though other studies demonstrate that, during this stage, there is an increase of lipid oxidation and oxidized proteins, as well as an increase in inflammation markers (Rivas-Arancibia et al., 2010; RodríguezMartínez et al., 2013).

The second stage corresponds to the group exposed to ozone for 30 days, and it is defined by the onset of A $\beta$ 1-42 intracellular immunoreactivity. It is spectrally accompanied by a significant growth of the sub-bands associated to unordered structures and $\beta$-turn, as well as an additional decrease of the relative abundance of the $\alpha$-helix secondary structure. Along with the previous changes, there is also a greater increase of oxidized lipids and proteins. In our model, these results indicate that the alterations are irreversible.

The third stage corresponds to the groups exposed for 60 and 90 days. In which, we observe an additional increase of immunoreactivity, both intracellularly and at the neuropil, together with cell swelling and pyknotic nuclei. This is spectrally associated with the emergence of a new predominant sub-band corresponding to the $\beta$-sheet secondary structure motif together with an additional reduction in the abundance of the sub-bands of $\alpha$-helix, and unordered and $\beta$-turn motifs. In this stage, the reduction of the $\beta$-turn and $\alpha$-helix structures seems to precede the formation of the $\beta$-sheet motifs, the predominant secondary structure of $\mathrm{A} \beta$ $1-42$.

These results are in agreement with general conclusions already established by other works, which used different methodologies and techniques (Soto et al., 1995; Kirkitadze et al., 2001), indicating that the A $\beta$ 1-42 original $\alpha$-helical conformation transforms into $\beta$-sheet, which finally results in the fibrillar $A \beta$ 1-42. Our results also indicate that the $A \beta$ peptide first forms a transient $\alpha$-helical conformation and then, when the neurodegeneration process progresses, there is an increase in the $\beta$-sheet conformation which is characteristic of $A \beta 1-42$ fibrillar. In this framework, the major contribution of this work is to provide a deeper understanding of the evolution of the structural motifs that shape the secondary structure of $\mathrm{A} \beta$ 1-42 from the original $\alpha$-helical conformation to the $\beta$-sheet structures. The results found in this work are summarized in Figure 6, which presents the hypothetical schematic representation of the conformational changes of A $\beta$ 1-42 peptide in hippocampal tissue of rats exposed to low ozone doses, this figure was bases 


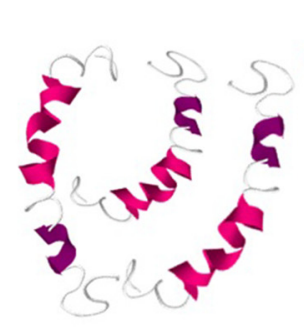

$A \beta$

$\alpha$-helix

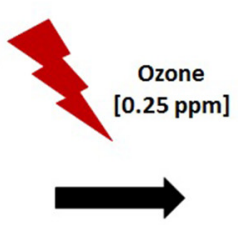

7, 15,30 exposure days

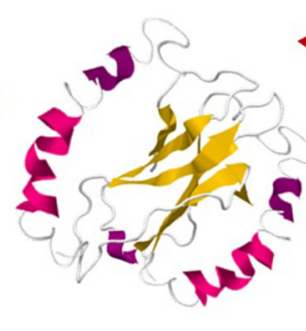

$A \beta_{1-42}$

$\alpha$-helix, $\beta$-turn, random coil

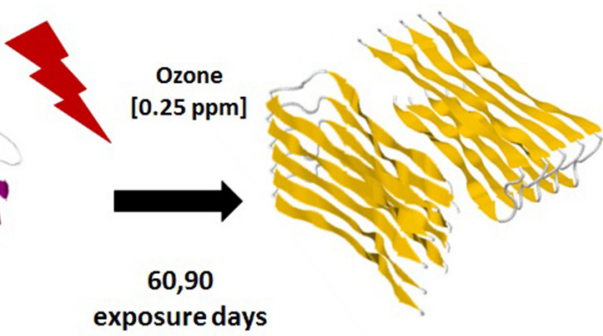

$A \beta_{1-42}$

$\beta$-sheet

progress exposure/conformational change/toxicity

FIGURE 6 | Hypothetical schematic representation of conformational changes of A $\beta$ 1-42 along ozone exposure. In the first stage, there is an abundance of the $\alpha$-helix conformation. After 30 days of ozone exposure, in a second stage, there is a mixture of $\alpha$-helix, $\beta$-turn and random coil motifs. After 90 days of ozone exposure, the third stage, there is a majority of $\beta$-sheet secondary structure conformation. This trend is related with the increase toxicity of ozone and the neurodegeneration progress in the hippocampus of rats. Model figures $1 \mathrm{ZOQ}, 2 \mathrm{OTK}$ and 2BEG, taken from the PDB files.

on the published structures obtained from different techniques (Lührs et al., 2005; Tomaselli et al., 2006; Hoyer et al., 2008).

On the other hand, there exists a clear process of progressive neurodegeneration followed by a major increase of oxidized biomolecules, mitochondrial deficit and a decrease of antioxidant systems (Rodríguez-Martínez et al., 2013). The above may indicate that, unlike $A \beta 1-40$, which is commonly formed in the brain (Yin et al., 2007), A $\beta$ 1-42 shows a change in its spatial conformation, promoted by oxidative stress (Butterfield et al., 2013; Korshavn et al., 2017). A $\beta$ 1-42 is an oxidized peptide that changes its spatial conformation from $\beta$-turn to $\beta$-sheet as proven by this work. This peptide may act as a reactive species since it leads to the oxidation of neighboring molecules as many other researchers have demonstrated (Butterfield et al., 2013). This indicates that the role of oxidative stress is fundamental in $A \beta$ 1-42 formation and during the maintenance of the process of progressive neurodegeneration. The intracellular increase of $\mathrm{A} \beta 1-42$ as well as its entry to the mitochondrion (Hernández-Zimbrón and Rivas-Arancibia, 2015) helps keeping the damage since it inhibits ATP production, which is necessary to repair and maintain cell metabolism (Rodríguez-Martínez et al., 2013). It also hampers the correct function of the endoplasmic reticulum as neurodegeneration progresses and leads to an increase of protein misfolding, which contributes to accumulate intracellular oxidized proteins leading to cell death (Rodríguez-Martínez et al., 2016).

Something similar may be occurring in $\mathrm{AD}$ since its diagnosis is done when the disease is advanced. Similarly, A $\beta$ 1-42 plaques seem to be the final outcome of a long process of pathophysiological changes and increase in the production of reactive species (Butterfield et al., 2013). These species work as signals to attract phagocytic microglia cells (RivasArancibia et al., 2010), macrophages and others, increasing the oxidative stress. Although several studies have shown that there are alterations in $\mathrm{A} \beta$ 1-42 fibers, the mechanisms through which $A \beta 1-42$ is progressively formed in the brain are still unclear. This study allows us to demonstrate how the conformation of the peptide progressively changes from $\alpha$-helix to $\beta$-turn plus unordered structure, and finally to $\beta$ sheet secondary structure. This might explain why inhibiting the formation of $A \beta$ 1-42 plaques cannot restore the normal functioning of the brain tissue and that it is necessary to counter the factors that contribute to neuronal damage, like oxidative stress.

\section{CONCLUSIONS}

The formation of $\mathrm{A} \beta \mathrm{1-42}$ is promoted in a murine model of progressive neurodegeneration similar to $\mathrm{AD}$, caused by repeated exposure to low doses of ozone, which induces a state of chronic oxidative stress. This peptide shows conformational changes from $\alpha$-helix to a mixture of $\alpha$-helix, $\beta$-turn and unordered structure, leading to a $\beta$-sheet secondary structure. This promotes polymerization, misfolding and protofibrill aggregation of $\mathrm{A} \beta$ 1-42 in neurotoxic and insoluble plaques. These conformational changes in the secondary peptide structures may be the key to understand the formation of both intra and extracellular $\mathrm{A} \beta$ 1-42 deposits, similar to what could be happening in the brain of $\mathrm{AD}$ patients.

\section{AUTHOR CONTRIBUTIONS}

SR-A proposed the objective of this work, designed the experimental strategy, contributed with the analysis, interpretation and discussion of the results obtained for this 
study and work on the writing of the manuscript. ER-M, carried out the techniques of immunohistochemistry, did Figure 2, contributed with the analysis, interpretation and discussion of the results obtained for this study and work on the writing of the manuscript. IB-R, carried out the Raman records, the analysis of the same and the realization of Figure 3 and Table 1, contributed with the analysis, interpretation and discussion of the results obtained for this study and work in the writing of the manuscript. UL-G, participated in the realization of Raman records, the analysis of the same and the realization of Figure 3. He contributed with the analysis, interpretation and discussion of the results obtained for this study and work on the writing of the manuscript. JMS participated in

\section{REFERENCES}

Ahmed, M., Davis, J., Aucoin, D., Sato, T., Ahuja, S., Aimoto, S., et al. (2010). Structural conversion of neurotoxic amyloid- $\beta_{(1-42)}$ oligomers to fibrils. Nat. Struct. Mol. Biol. 17, 561-567. doi: 10.1038/nsmb.1799

Barth, A., and Zscherp, C. (2002). What vibrations tell us about proteins. Q. Rev. Biophys. 35, 369-430. doi: 10.1017/S0033583502003815

Block, M. L., and Calderón-Garcidueñas, L. (2009). Air pollution: mechanisms of neuroinflammation and CNS disease. Trends Neurosci. 32, 506-516. doi: 10.1016/j.tins.2009.05.009

Bonnier, F., and Byrne, H. J. (2012). Understanding the molecular information contained in principal component analysis of vibrational spectra of biological systems. Analyst 137, 322-332. doi: 10.1039/clan15821j

Boyd-Kimball, D., Sultana, R., Mohmmad-Abdul, H., and Butterfield, D. A. (2004). Rodent $A \beta(1-42)$ exhibits oxidative stress properties similar to those of human $\mathrm{A} \beta(1-42)$ : implications for proposed mechanisms of toxicity. J. Alzheimers Dis. 6, 515-525. doi: 10.3233/jad-2004-6509

Buchete, N. V., Tycko, R., and Hummer, G. (2005). Molecular dynamics simulations of Alzheimer's $\beta$-amyloid protofilaments. J. Mol. Biol. 353, 804-821. doi: 10.1016/j.jmb.2005.08.066

Butterfield, D. A., Swomley, A. M., and Sultana, R. (2013). Amyloid $\beta$-peptide (1-42)-induced oxidative stress in Alzheimer disease: importance in disease pathogenesis and progression. Antioxid. Redox Signal. 19, 823-835. doi: $10.1089 /$ ars.2012.5027

Chen, P., Shen, A., Zhao, W., Baek, S. J., Yuan, H., and Hu, J. (2009). Raman signature from brain hippocampus could aid Alzheimer's disease diagnosis. Appl. Opt. 48, 4743-4748. doi: 10.1364/ao.48.004743

Dorado-Martínez, C., Paredes-Carbajal, C., Mascher, D., Borgonio-Pérez, G., and Rivas-Arancibia, S. (2001). Effects of different ozone doses on memory, motor activity and lipid peroxidation levels, in rats. Int. J. Neurosci. 108, 149-161. doi: 10.3109/00207450108986511

Friedemann, M., Helk, E., Tiiman, A., Zovo, K., Palumaa, P., and Tõugu, V. (2015). Effect of methionine-35 oxidation on the aggregation of amyloid$\beta$ peptide. Biochem. Biophys. Rep. 3, 94-99. doi: 10.1016/j.bbrep.2015. 07.017

Hernández-Zimbrón, L. F., and Rivas-Arancibia, S. (2014). Deciphering an interplay of proteins associated with amyloid $\beta$ 1-42 peptide and molecular mechanisms of Alzheimer's disease. Rev. Neurosci. 25, 773-783. doi: 10.1515/revneuro-2014-0025

Hernández-Zimbrón, L. F., and Rivas-Arancibia, S. (2015). Oxidative stress caused by ozone exposure induces $\beta$-amyloid 1-42 overproduction and mitochondrial accumulation by activating the amyloidogenic pathway. Neuroscience 304, 340-348. doi: 10.1016/j.neuroscience.2015.07.011

Hoyer, W., Grönwall, C., Jonsson, A., Ståhl, S., and Hárd, T. (2008). Stabilization of a $\beta$-hairpin in monomeric Alzheimer's amyloid- $\beta$ peptide inhibits amyloid formation. Proc. Natl. Acad. Sci. U S A 105, 5099-5104. doi: 10.1073/pnas. 0711731105

Jung, C. R., Lin, Y. T., and Hwang, B. F. (2015). Ozone, particulate matter and newly diagnosed Alzheimer's disease: a population-based cohort the design of the experimental strategy, contributed with the analysis, interpretation and discussion of the Raman results obtained for this study and work on the writing of the manuscript.

\section{ACKNOWLEDGMENTS}

The authors thank Gabino Borgonio-Pérez for his technical support and the Laboratorio Universitario de Caracterización Espectroscópica for its technical support with the Raman spectroscopy studies. This work was supported by Consejo Nacional de Ciencia y Tecnologia (CONACyT) (grant 219703) to SR-A. study in Taiwan. J. Alzheimers Dis. 44, 573-584. doi: 10.3233/JAD140855

Kirkitadze, M. D., Condron, M. M., and Teplow, D. B. (2001). Identification and characterization of key kinetic intermediates in amyloid $\beta$-protein fi brillogenesis. J. Mol. Biol. 312, 1103-1119. doi: 10.1006/jmbi.2001.4970

Kong, J., and Yu, S. (2007). Fourier transform infrared spectroscopic analysis of protein secondary structures. Acta Biochim. Biophys. Sin. (Shanghai) 39, 549-559. doi: 10.1111/j.1745-7270.2007.00320.x

Korshavn, K. J., Satriano, C., Lin, Y., Zhang, R., Dulchavsky, M., Bhunia, A., et al. (2017). Reduced lipid bilayer thickness regulates the aggregation and cytotoxicity of amyloid- $\beta$. J. Biol. Chem. 292, 4638-4650. doi: 10.1074/jbc. M116.764092

Kurouski, D., Van Duyne, R. P., and Lednev, I. K. (2015). Exploring the structure and formation mechanism of amyloid fibrils by Raman spectroscopy: a review. Analyst 140, 4967-4980. doi: 10.1039/c5an00342c

Lee, M., Chang, H. J., Park, J. Y., Shin, J., Park, J. W., Choi, J. W., et al. (2016). Conformational changes of A $\beta$ (1-42) monomers in different solvents. J. Mol. Graph. Model. 65, 8-14. doi: 10.1016/j.jmgm.2016.02.003

Lippert, J. L., Tyminski, D., and Desmeules, P. J. (1976). Determination of the secondary structure of proteins by laser Raman spectroscopy. J. Am. Chem. Soc. 98, 7075-7080. doi: 10.1021/ja00438a057

Lührs, T., Ritter, C., Adrian, M., Riek-Loher, D., Bohrmann, B., Döbeli, H., et al. (2005). 3D structure of Alzheimer's amyloid- $\beta(1-42)$ fibrils. Proc. Natl. Acad. Sci. U S A 102, 17342-17347. doi: 10.1073/pnas.0506723102

Mahadevan-Jansen, A., and Richards-Kortum, R. (1996). Ramanspectroscopy for the detection of cancers and precancers. J. Biomed. Opt. 1, 31-70. doi: $10.1117 / 12.227815$

Moulton, P. V., and Yang, W. (2012). Air pollution, oxidative stress, and Alzheimer's disease. J. Environ. Public Health 2012, 4727-4751. doi: 10.1155/2012/472751

Movasaghi, Z., Rehman, S., and Rehman, I. U. (2007). Raman spectroscopy of biological tissues. Appl. Spectrosc. Rev. 42, 493-541. doi: 10.1080/05704920701551530

Mumaw, C. L., Levesque, S., McGraw, C., Robertson, S., Lucas, S., Stafflinger, J. E., et al. (2016). Microglial priming through the lung-brain axis: the role of air pollution-induced circulating factors. FASEB J. 30, 1880-1891. doi: 10.1096/fj. 201500047

Park, A., Baek, S. J., Shen, A., and Hu, J. (2013). Detection of Alzheimer's disease by Raman spectra of rat's platelet with a simple feature selection. Chemometr. Intell. Lab. Syst. 121, 52-56. doi: 10.1016/j.chemolab.2012.11.011

Pereyra-Muñoz, N., Rugerio-Vargas, C., Angoa-Pérez, M., Borgonio-Pérez, G., and Rivas-Arancibia, S. (2006). Oxidative damage in substantianigra and striatum of rats chronically exposed to ozone. J. Chem. Neuroanat. 31, 114-123. doi: 10.1016/j.jchemneu.2005.09.006

Rahmelow, K., Hübner, W., and Ackermann, T. (1998). Infrared absorbances of protein side chains. Anal. Biochem. 257, 1-11. doi: 10.1006/abio.1997.2502

Rivas Arancibia, S., Gallegos-Ríos, C., Gomez-Crisostomo, N., FerreiraGarcidueñas, E., Briseño, D. F., Navarro, L., et al. (2011). "Oxidative stress and neudodegenerative disease," in Neurodegenerative Diseases-Processes, 
Prevention, Protection and Monitoring, ed. R. C. C. Chang (Rijeka: Intech.), 113, 53-88. doi: 10.5772/1252

Rivas-Arancibia, S., Guevara-Guzmán, R., López-Vidal, Y., RodríguezMartínez, E., Zanardo-Gomes, M., Angoa-Pérez, M., et al. (2010). Oxidative stress caused by ozone exposure induces loss of brain repair in the hippocampus of adult rats. Toxicol. Sci. 113, 187-197. doi: 10.1093/toxsci/ kfp252

Rivas-Arancibia, S., Vazquez-Sandoval, R., Gonzalez-Kladiano, D., SchneiderRivas, S., and Lechuga-Guerrero, A. (1998). Effects of ozone exposure in rats on memory and levels of brain and pulmonary superoxide dismutase. Environ. Res. 76, 33-39. doi: 10.1006/enrs.1997.3784

Rodríguez-Martínez, E., Martínez, F., Espinosa-García, M. T., Maldonado, P., and Rivas-Arancibia, S. (2013). Mitochondrial dysfunction in the hippocampus of rats caused by chronic oxidative stress. Neuroscience 252, 384-395. doi: 10.1016/j.neuroscience.2013.08.018

Rodríguez-Martínez, E., Nava-Ruiz, C., Escamilla-Chimal, E., Borgonio-Perez, G., and Rivas-Arancibia, S. (2016). The effect of chronic ozone exposure on the activation of endoplasmic reticulum stress and apoptosis in rat hippocampus. Front. Aging Neurosci. 8:245. doi: 10.3389/fnagi.2016.00245

Ruiz-Chica, A. J., Medina, M. A., Sanchez-Jimenez, F., and Ramirez, F. J. (2004). Characterization by Raman spectroscopy of conformational changes on guaninecytosine and adenine-thymine oligonucleotides induced by aminooxyanalogues of spermidine. J. Raman Spectrosc. 35, 93-100. doi: $10.1002 /$ jrs. 1107

Rygula, A., Majzner, K., Marzec, K. M., Kaczor, A., Pilarczyk, M., and Baranska, M. (2013). Raman spectroscopy of proteins: a review. J. Raman Spectrosc. 44, 1061-1076. doi: 10.1002/jrs.4335

Sekhar, A. C., Movasaghi, Z., Rehman, S., and Rehman, I. U. (2015). Raman spectroscopy of biological tissues. Appl. Spectrosc. Rev. 50, 46-111. doi: 10.1080/05704928.2014.923902

Soto, C., Castano, E. M., Frangione, B., and Inestrosa, N. C. (1995). The $\alpha$-helical to $\beta$-strand transition in the amino-terminal fragment of the amyloid $\beta$-peptide modulates amyloid fibril formation. J. Biol. Chem. 270, 3063-3067. doi: 10.1074/jbc.270.7.3063

Sudworth, C. D., and Krasner, N. (2004). Raman spectroscopy of Alzheimer's diseased tissue. Proc. SPIE 5321, 93-101. doi: 10.1117/12. 552869

Tay, L. L., Tremblay, R. G., Hulse, J., Zurakowski, B., Thompson, M., and Bani-Yaghoub, M. (2011). Detection of acute brain injury by Raman spectral signature. Analyst 136, 1620-1626. doi: 10.1039/c0an00897d
Tomaselli, S., Esposito, V., Vangone, P., van Nuland, N. A., Bonvin, A. M., Guerrini, R., et al. (2006). The $\alpha$-to- $\beta$ conformational transition of Alzheimer's $\mathrm{A} \beta-(1-42)$ peptide in aqueous media is reversible: a step by step conformational analysis suggests the location of $\beta$ conformation seeding. Chembiochem 7 , 257-267. doi: 10.1002/cbic.200500223

Uzunbajakava, N., Lenferink, A., Kraan, Y., Willekens, B., Vrensen, G., Greve, J., et al. (2003). Nonresonant Raman imaging of protein distribution in single human cells. Biopolymers 72, 1-9. doi: 10.1002/bip.10246

Watson, A. A., Fairlie, D. P., and Craik, D. J. (1998). Solution structure of methionine-oxidized amyloid $\beta$-peptide (1-40). Does oxidation affect conformational switching? Biochemistry 37, 12700-12706. doi: $10.2210 / \mathrm{pdb} 1 \mathrm{ba} / \mathrm{pdb}$

Wen, Z. Q. (2007). Raman spectroscopy of protein pharmaceuticals. J. Pharm. Sci. 96, 2861-2878. doi: 10.1002/jps.20895

Xiao, Y., Ma, B., McElheny, D., Parthasarathy, S., Long, F., Hoshi, M., et al. (2015). $A \beta(1-42)$ fibril structure illuminates self-recognition and replication of amyloid in Alzheimer's disease. Nat Struct Mol Biol. 6, 499-505. doi: $10.1038 / \mathrm{nsmb} .2991$

Yin, Y. I., Bassit, B., Zhu, L., Yang, X., Wang, C., and Li, Y. M. (2007). $\gamma$-Secretase substrate concentration modulates the A $\beta 42 / \mathrm{A} \beta 40$. J. Biol. Chem. 282, 23639-23644. doi: 10.1074/jbc.M704601200

Zhang, S., Iwata, K., Lachenmann, M. J., Peng, J. W., Li, S., Stimson, E. R., et al. (2000). The Alzheimer's peptide $A \beta$ adopts a collapsed coil structure in water. J. Struct. Biol. 130, 130-141. doi: 10.1006/jsbi.2000.4288

Zhu, X., Bora, R. P., Barman, A., Singh, R., and Prabhakar, R. (2012). Dimerization of the full-length Alzheimer amyloid $\beta$-peptide $(\mathrm{A} \beta 42)$ in explicit aqueous solution: a molecular dynamics study. J. Phys. Chem. B 116, 4405-4416. doi: $10.1021 /$ jp210019h

Conflict of Interest Statement: The authors declare that the research was conducted in the absence of any commercial or financial relationships that could be construed as a potential conflict of interest.

Copyright (c) 2017 Rivas-Arancibia, Rodríguez-Martínez, Badillo-Ramírez, López-González and Saniger. This is an open-access article distributed under the terms of the Creative Commons Attribution License (CC BY). The use, distribution or reproduction in other forums is permitted, provided the original author(s) or licensor are credited and that the original publication in this journal is cited, in accordance with accepted academic practice. No use, distribution or reproduction is permitted which does not comply with these terms. 